\title{
Smart Phone For Personal Health Care MONITORING SYSTEM
}

\author{
Chayalakshmi C.L. \\ Department of Electronics and Instrumentation Engineering, \\ Basaveshwar Engineering College (Autonomous), Bagalkot, Karnataka, India
}

\begin{abstract}
:
Health is of great concern for everyone. Both investment and efforts on health monitoring, care and maintenance are ever increasing. Smart phones are affordable for every one nowadays.

This paper discusses an innovative method ofdesigninga smart phone based health care system which can measure some of the body parameters such as temperature, pulse rate and blood glucose level. The key feature here is to embed the smart phone with required sensors, process their data, store in the memory. Further, these data can be transmitted to a doctor or hospital authority for future references using an application designed in the same smart phone. The system can be used to suggest some precautions and also remedies for the user, if these body parameters are outside the normal range. This makes the smart phone really the smartest.
\end{abstract}

\section{INTRODUCTION:}

Health is wealth. Health is a state of complete physical, mental and social wellbeing, and not merely the absence of disease or infirmity. Anything which disturbs healthy body is disease. A disease is a particular abnormal, pathological condition that affects part or all of an organism. It is a medical condition associated with specific symptoms and signs [1]. As the population is increasing rapidly day by day, in the same way death rate is also increasing due to diseases at a faster rate. Common abnormalities in human body parameters leading to serious conditions are blood glucose level, blood pressure, varying body temperature and so on.

Heart rate, or heart pulse, is the speed of the heartbeat measured by the number of heartbeats per unit of time - typically beats per minute (bpm) [2]. Normal human body temperature, also known as normothermia or euthermia, depends upon the place in the body at which the measurement is made, the time of day, as well as the activity level of the person [3]. Diabetes, a group of metabolic diseases in which the person has high blood glucose (blood sugar), either because insulin production is inadequate or because of the body cells does not respond properly to insulin, or both [4]. 
International Journal Of Instrumentation And Control Systems (IJICS) Vol.6, No.4, October 2016

Table1 provides the number of people affected in millions because of the diabetes[5].

\begin{tabular}{|c|c|}
\hline Year & Number of people (in millions) \\
\hline 1995 & 124.7 \\
\hline 2005 & 153.9 \\
\hline 2025 & 299.1 \\
\hline
\end{tabular}

\section{Table 1: Number Of People Affected}

Monitoring and controlling these parameters is very essential for a healthy life. If they are not controlled at a particular stage, it may lead to severe problems and affect different body parts, leading to a complication that can affect any organ in the body, such as heart, loss of vision, failure of kidney, nervous break-down or gum and teeth.

Heart disease and blood vessel disease are the biggest complications that people with uncontrolled diabetes face. In 2004, approximately $68 \%$ of diabetes-related death certificates among people aged 65 years or older were related to heart disease, with stroke being noted in 16 $\%$ of death certificates. Diabetes can also cause poor blood flow in the legs and feet (peripheral artery disease). Adults with diabetes have heart disease death rates about two to four times higher than adults without diabetes. The risk for stroke is two to four times higher. Diabetes is the leading cause of new blindness in the U.S. in adults ages 20-74. It can cause a number of eye problems, some of which can lead to blindness if not addressed. The eye disorders include: Glaucoma, Cataract, Diabetic retinopathy. Over time, high blood sugar levels can harm the nerves. This can lead to loss of sensation or feeling (usually starting in the toes) or pain and burning of the feet. Approximately $60-70 \%$ of people with diabetes have some form of nerve damage.

An individual with a high temperature initiated by an infection should have a limit in the communication to others for the prevention of spreading. Uncontrolled high blood pressure can damage heart in a number of ways, such as chest pain, a heart attack or irregular heart rhythms (arrhythmias). High blood pressure forces heart to work harder than necessary in order to pump blood to the rest of the body. This causes the left ventricle to thicken or stiffen (left ventricular hypertrophy).Eventually, heart simply begins to wear out and fail. Uncontrolled high blood pressure can lead to stroke by damaging and weakening brain's blood vessels, causing them to narrow, rupture or leak. High blood pressure is one of the most common causes of kidney failure. That is because it can damage both the large arteries leading to kidneys and the tiny blood vessels (glomeruli) within the kidneys. Occasionally, a low body temperature can indicate an underlying problem or condition, such as: Underactive thyroid (hypothyroidism), Adrenal gland insufficiency (Addison's disease), Hypothalamus dysfunction.

So to avoid these above mentioned problems in further stages, it is very important to measure these essential parameters and control them. Parameters like blood glucose level, blood pressure, body temperature can be measured using Micro-Electro-Mechanical Systems (MEMS) sensors. MEMS is a technology that in its most general form can be defined as miniaturized mechanical and electro-mechanical elements (i.e., devices and structures) that are made using the techniques of micro-fabrication [6].Mobile technologies are increasingly used in Primary Health Care Services (PHCS). Use of Mobile Devices ensures improved access to primary healthcare and its 
gate-keeping function leads to less hospitalization, and less chance of patients being subjected to inappropriate growing in developing countries like India.The availability of low-cost mobile phones and the already broad coverage of GSM networks in India is a huge opportunity to provide services that would trigger development and improve people's lives[7].GSM is abbreviated as Global System for Mobile Communication. GSM modem has a slot for inserting SIM (Subscriber Identity Module). GSM network contains Mobile Station, Base station subsystem and Network subsystem. Subhani et al. have discussed a method of GSM based heart rate and temperature monitoring system which can be more suitable for practical implementation in India[8].

Many types of chronic illness, such as diabetes, abnormal blood pressure are essentially required to be monitored continuously. In particular, self-monitoring is important for heightened awareness and progress tracking[9], [10].

A personal health monitoring system is proposed in this paper and through this proposed system, every individual is able to self-monitor vital body parameters, consult doctor and get remedies from the doctor without direct approach. This is possible using GSM and it reduces time consumption and cost of hospitalization. Besides, the problem of the lack of unremitted real-time care for every patient, which is caused by the shortage of health care members, is also solved.

\section{Block Diagram And Working PrinCiPle:}

The block diagram of the proposedsystem is shown in Fig.1.A particular app is to be installed in a smartphone. That application is specially designed for monitoring and displaying human body parameters which are further transmitted to doctor's cell phone using GSM network. When the installed app is opened, it has an option of selecting particular parameter as per user's desire.The parameters to be measured are: body temperature (using MEMS sensor LM35), pulse rate(using IR LED and detector),blood glucose (using MEMs viscometric sensor).LM35 temperature sensor is used to measure the body temperature. This sensor unit works under low power DC input of $5 \mathrm{~V}$. When the MEMs sensor comes in contact with skin surface, it measures the temperature and sends data to signal conditioning block. Pulse rate is detected with IR LED and LDR, which are placed parallel to each other. LED emits IR (Infrared) rays so that, when the finger is placed in between LED and LDR arrangement, so that there exists some systolic pressure. LED emits IR rays which are travelled through finger and blood flows with arteriole pressure. Whenever systolic pressure is applied, normal pressure of blood flow is disturbed at fingertip which is high and IR rays penetrate through blood and are received by LDR which are sent to signal conditioning block.

Various methods are available for glucose sensing. Glucose sensing based on affinity binding principles using a solution of dextran Concanavalin-A (Con A) as the sensing fluid is more suitable for our application [11].

The glucose concentration is determined by detecting viscosity changes induced by the binding of glucose to Con A through the measurement of the cantilever's vibration parameters. The output of sensor/detector is further given to signal conditioning block.A mobile processor can be throttled down to different power levels or sections of the chip can be turned off entirely when not in use. Further, the clock frequency may be stepped down under low processor loads. This stepping 
down conserves power and prolongs battery life. Output of signal conditioning block is sent to processor where it is store in its memory. Normally Advanced RiscMachine (ARM) processor is suitable for this application. The data processed and stored in memory of mobile processor is compared with already saved data and ranges. That data is further transferred to display on screen.

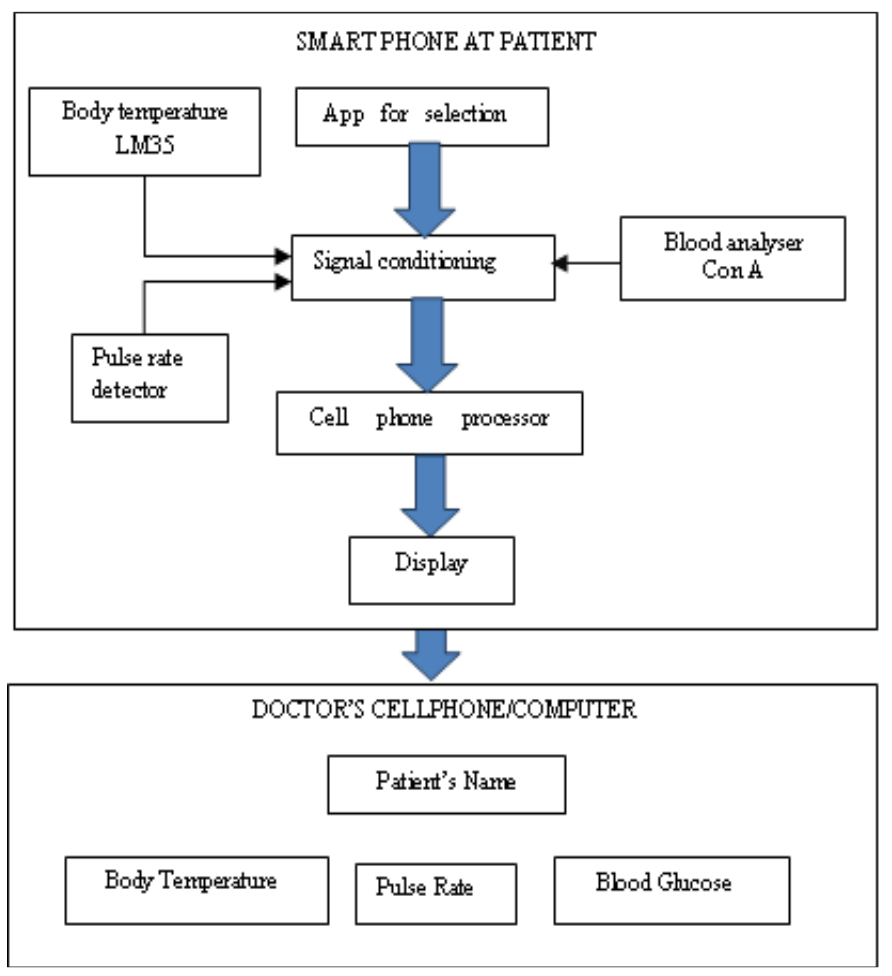

Fig. 1: Block diagram of the system

Themeasured parameter from MEMs sensors are compared with the data base available in the processor of smart-phone. A data base for body temperature, pulse rate, blood glucose, is created in the smart-phone based on the age, whether the person is a male/female or athlete. After comparison the processor must take appropriate decision to display the parameter as normal or abnormal, if abnormal they are sent to doctor's cell phone via GSM network. For controlling the abnormalities, the smart-phone displays the type of exercises or required diet on screen.

\section{SOFTWARE DETAILS:}

The algorithm required for the system is shown in Fig.2. For the measurement of any biological parameter first the application is to be selected. Then the user should select the appropriate path for the measurement of either body temperature, blood glucose level or pulse rate measurement. In this flow chart path A is for measuring the body temperature, path B is for measuring blood glucose level and path $\mathrm{C}$ is for measuring the pulse rate. 
When the first parameter is selected by the user, the body temperature is measured with the help of LM35. The output from this sensor is $10 \mathrm{mV} /{ }^{\circ} \mathrm{C}$ and this voltage is amplified with the help of signal conditioning circuit. The amplified output from LM35 is sent to the cell phone processor. The processor then converts the data into body temperature in terms of degree Celsius and the same is compared with the minimum and the maximum range for a healthy person. If the measured value is within the range then the body temperature is displayed on the screen. If the measured value is out of range, then the processor displays the probable remedies along with displaying the body temperature and the information is sent to the family doctor using GSM module.

If the user selects path B, then the arrangement has to be made in the smart phone in such a way that the notch having a small needle moves outside the smart phone to collect the blood sample.

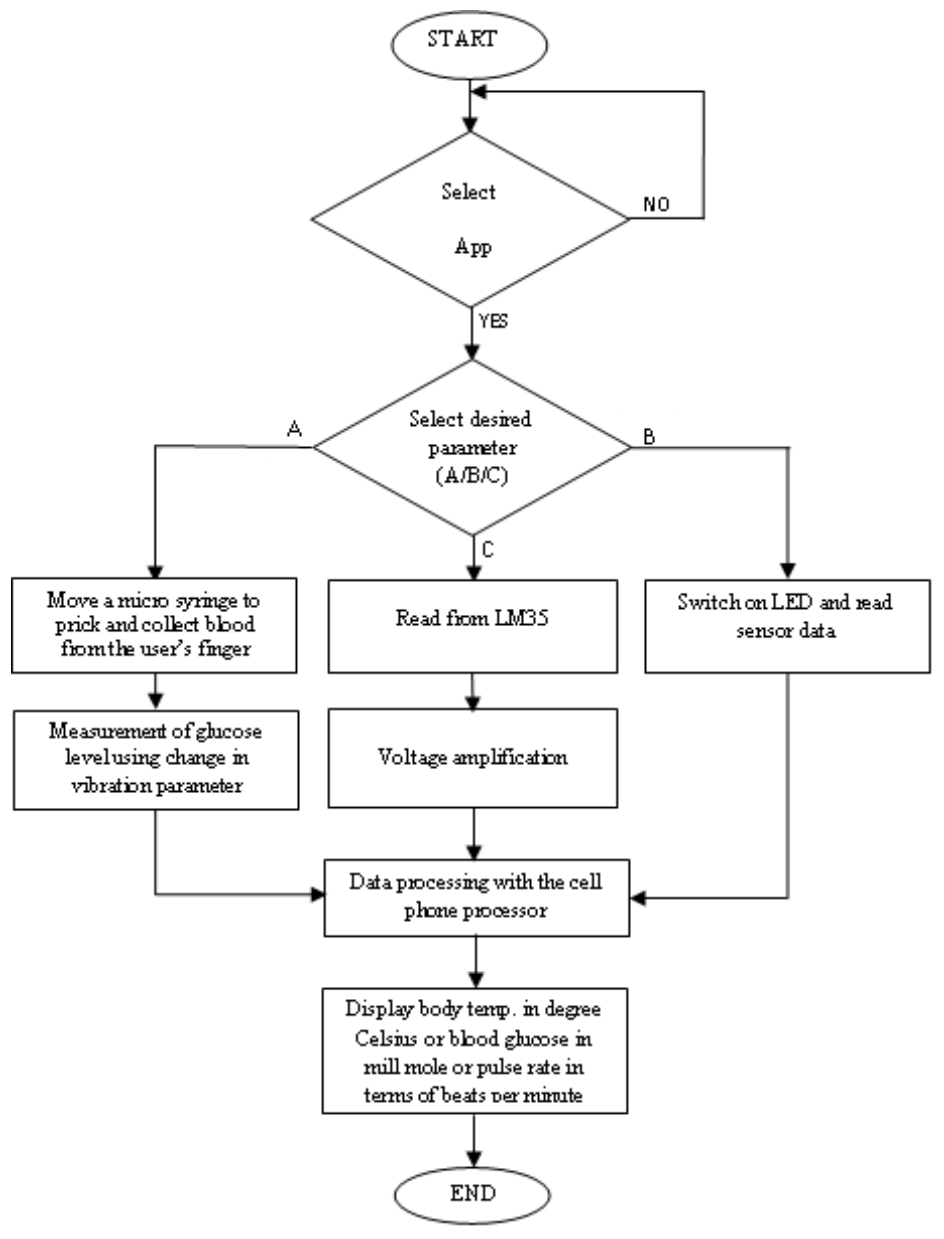

Fig. 2: Flow chart for the process

The glucose level is measured using the change in the vibration of cantilever beam in the sensor. The sensor output is further provided to the processor of smart phone to convert change in vibration to blood glucose level in terms of milli-moles. The processor then compares the measured blood glucose level with the minimum and maximum range for a healthy person. If the 
measured value is within the range then the processor displays the blood glucose level. If the measured value is outside the range then the processor provides suggestions to the user along with displaying the measured value of screen. Finally the same information is transmitted to the cell phone or computer of family doctor using GSM module.

If the user selects path C, then the IR LED is switched on which is situated between the fingers of a user when-ever the user holds the smart phone. The IR detector which is placed parallel to the IR LED collects the pulses and provides the number of pulses to the smart phone processor. The counter of smart phone processor counts the number of pulses obtained from the IR detector for one complete minute. Then the counter value is compared with the minimum and maximum range of pulse rate for a healthy person. If the measured value is within the range the pulse rate in terms of number of beats per minute is displayed. If the measured pulse rate is outside the range, then the processor displays suggestions along with displaying the measured value.

\section{CONCLUSION:}

A smart phone based system for measuring and monitoring the body parameters by creating a unique application is discussed in this paper. By sitting at home one can know his/her body parameters. Facility is also provided to transmit the information to the family doctor in the abnormal condition, so that family doctor is also informed with the patient's condition. If required or in emergency situation, doctor can also suggest remedies to the patient before visiting him/her.

\section{REFERENCES:}

[1] https://en.wikipedia.org/wiki/Disease

[2] www.webmd.com/heart-disease/pulse-measurement

[3] https://en.wikipedia.org/wiki/Human_body_temperature

[4] https://en.wikipedia.org/wiki/Diabetes_mellitus

[5] www.diabetesindia.com/diabetes/itfdci.htm

[6] https://www.mems-exchange.org/MEMS/what-is.htm

[7] Darrell West,"Managing Chronic Diseases Through Remote Monitoring and Mobile Devices”,CENTRE FOR INNOVATION TECHNOLOGY AT BROOKINGS,May 2012

[8] Subhani S. K. M., Sateesh G.N.V, Chaitanya Ch., Prakash Babu G, "Implementation of GSM based heart rate and temperature monitoring system", Research Journal of Engineering Sciences, Vol. 2 (4), pp 43-45, April 2013.

[9] Guanling Chen, Bo Yan,MinhoShin,DavidKotz, Ethan Berke, “MPCS: Mobile-Phone Based Patient Compliance System for Chronic Illness Care', The Policy Journal of the Health Sphere, 20(6), 2001.

[10] E. H. Wagner, B. T. Austin, C. Davis, M. Hindmarsh, J. Schaefer, and A. Bonomi. Improving chronic illness care: Translating evidence into action.

[11] Yongjun Zhao, SiqiLi,ArthurDavidson,BozhiYang,Qian Wang, Qiao Lin, "A MEMS viscometric sensor for continuous glucose monitoring", Journal of Micromechanics andMicroengineering, 14 November 2007. 
International Journal Of Instrumentation And Control Systems (IJICS) Vol.6, No.4, October 2016 